\title{
Article \\ Comparative Microbiomes of the Respiratory Tract and Joints of Feedlot Cattle Mortalities
}

\author{
Chunli $\mathrm{Li}^{1,+}{ }^{1}$, Rahat Zaheer $\left.{ }^{1,+}{ }^{(}\right)$, Andrea Kinnear ${ }^{2}{ }^{-}$, Murray Jelinski ${ }^{2}{ }^{(0)}$ and Tim A. McAllister ${ }^{1, *(1)}$ \\ 1 Lethbridge Research and Development Centre, Agriculture and Agri-Food Canada, \\ Lethbridge, AB T1J 4B1, Canada; lichunli_1978@hotmail.com (C.L.); rahat.zaheer@agr.gc.ca (R.Z.) \\ 2 Large Animal Clinical Sciences, Western College of Veterinary Medicine, University of Saskatchewan, \\ 52 Campus Drive, Saskatoon, SK S7N 5B4, Canada; andrea.kinnear@usask.ca (A.K.); \\ murray.jelinski@usask.ca (M.J.) \\ * Correspondence: tim.mcallister@agr.gc.ca; Tel.: +1-403-315-9916 \\ + These authors have equally contributed to this work.
}

Citation: Li, C.; Zaheer, R.; Kinnear, A.; Jelinski, M.; McAllister, T.A. Comparative Microbiomes of the Respiratory Tract and Joints of Feedlot Cattle Mortalities. Microorganisms 2022, 10, 134. https://doi.org/10.3390/ microorganisms10010134 Academic Editor: Benoit St Pierre Received: 15 December 2021 Accepted: 6 January 2022 Published: 10 January 2022 Publisher's Note: MDPI stays neutral with regard to jurisdictional claims in published maps and institutional affiliations.

Copyright: (c) 2022 by the authors. Licensee MDPI, Basel, Switzerland. This article is an open access article distributed under the terms and conditions of the Creative Commons Attribution (CC BY) license (https:/ / creativecommons.org/licenses/by/ $4.0 /)$.

\begin{abstract}
A comparative study of microbiota of the respiratory tract and joints of bovine respiratory disease (BRD) cattle mortalities was undertaken. Nasopharynx, trachea, lung and joint samples were collected from 32 cattle that died of BRD, "cases", and 8 that died of other causes, "controls". Bacterial diversity was lower $(p<0.05)$ in the nasopharynx, trachea and lungs of cases as compared to controls. In cases, alpha-diversity $(p<0.05)$ was lower in the lungs and joints than the nasopharynx. Proteobacteria, Tenericutes, Bacteroidetes, Firmicutes and Actinobacteria were the most abundant phyla in all samples. Relative abundances of Mycoplasma spp. in the lung, Pasteurella spp. in the trachea and lung, and Histophilus spp. in the lung, trachea and nasopharynx of cases were higher $(p<0.001)$ than controls. Mycoplasma spp. comprised $20.5 \%$ of bacterial flora in the joint, $36.0 \%$ in the lung, $22.4 \%$ in the trachea and $8.8 \%$ in the nasopharynx. Mannheimia spp. $(21.8 \%)$ and Histophilus spp. (10.4\%) were more abundant in lungs. Cattle that died of BRD possessed less diverse respiratory microbiomes with a higher abundance of respiratory pathogens. Mycoplasma spp. were prominent members of pneumonic lungs and joints displaying septic arthritis.
\end{abstract}

Keywords: bovine respiratory disease; arthritis; feedlot cattle; microbiome; nasopharynx; trachea; lung; joint

\section{Introduction}

Bovine respiratory disease (BRD) is the most significant cause of mortalities in newly arrived feedlot calves [1], costing the North American feedlot industry over \$4 billion annually due to treatment and prevention costs as well as lost productivity [2,3]. BRD is a multifactorial disease precipitated by various stressors (e.g., weaning, transportation, and commingling) that predispose cattle to viral and bacterial infections, with bacterial pathogens being considered the principle etiological agents. Primary bacterial pathogens associated with BRD include Pasteurella multocida, Mannheimia haemolytica, Histophilus somni and Mycoplasma bovis [4,5], all of which can colonize the upper respiratory tract as commensals in healthy cattle [6-8]. Presumably, when exposed to the appropriate combination of stressors, cattle become immunocompromised, leading to a proliferation of bacteria that colonize the lungs, resulting in pneumonia [6,9-11]. M. haemolytica (formerly Pasteurella haemolytica) infections in feedlot cattle are colloquially known as "Shipping fever" or "Pasteurellosis" and are characterized by a high fever and depression with postmortem findings of a severe, acute hemorrhagic fibrinonecrotic bronchopneumonia. P. multocida is associated with a fibrinopurulent bronchopneumonia, which results in less fibrin and necrosis and is hence less fulminant. H. somni is associated with purulent bronchopneumonia, severe fibrinous pleuritis, and a septicemia that may lead to secondary infections in other organs. Generally, P. multocida, M. haemolytica and H. somni are associated with 
BRD that develops within days to weeks of arrival at the feedlot. In contrast, pneumonia and polyarthritis cases associated with $M$. bovis tend to develop later in the feeding period [6,12-14]. In addition to colonizing the upper respiratory tract, hematologic dissemination of $M$. bovis from the lungs may lead to secondary colonization of joints and the development of polyarthritis [15].

A previous study reported that nasopharyngeal bacterial communities of cattle with BRD had lower diversity and richness as compared to their healthy counterparts [5]. Furthermore, the bacterial communities inhabiting the nasopharynx and trachea of healthy feedlot cattle were distinct from those associated with cattle suffering from bronchopneumonia [16]. Bacterial communities have also been shown to differ between the lower and upper respiratory tracts $[16,17]$. The microbiome of the trachea of feedlot cattle has a lower alpha diversity when compared to the nasopharynx, regardless of clinical status $[16,17]$. Although M. haemolytica, P. multocida, H. somni, and M. bovis are commonly isolated from the lower respiratory tract of pneumonic cattle, most microbiome studies have focused on the nasopharynx or trachea $[5,7,8,16,18-20]$.

The objective of this study was to compare bacterial communities of nasopharynx, trachea, lungs and joints from cattle that had lesions consistent with bovine respiratory disease ("cases") versus those that died of other non-BRD causes ("controls"). We hypothesized that bacterial communities would also differ by location within the respiratory system, and between the lungs and joints of cattle that died of BRD.

\section{Materials and Methods}

\subsection{Animals}

Samples were collected from beef cattle from September 2018 to December 2019 at four commercial feedlots in Southern Alberta, Canada. On arrival, all cattle were weighed (204 to $350 \mathrm{~kg}$ ) and received a metaphylaxis treatment of tulathromycin (Draxxin, $2.5 \mathrm{mg} \mathrm{kg}^{-1}$; Zoetis, Kalamazoo, MI, USA). Trained pen riders monitored the health of the cattle on a daily basis and removed those cattle displaying clinical signs of disease, of which BRD and lameness cases were most prevalent. Cattle were administered antimicrobials according to treatment protocols devised by the feedlot veterinarians. Tulathromycin was used as a treatment along with a variety of other antimicrobials including enrofloxacin (Baytril; Elanco Canada Ltd., Guelph, ON, Canada), ceftiofur (Ceftiocyl; Vetoquinol, Lavaltrie, QC, Excede or Excenel; Zoetis), florfenicol (Florkem; Ceva Animal Health Inc., Cambridge, ON, Canada), florfenicol/flunixin (Resflor; Merck Animal Health, Kirkland QC, Canada), oxytetracycline (Oxymycine LA 200, Oxymycine LA 300; Zoetis, Oxyvet 100 LP or Oxyvet 200 LA; Vetoquinol), and sulfadoxine/trimethoprim (Trimidox; Vetoquinol).

Cattle ( 300 head per pen) were housed in large $\left(8500 \mathrm{~m}^{2}\right)$ outdoor dirt-floor pens and were fed a balanced barley-based growing diet twice daily. Water was provided ad libitum and feed deliveries were adjusted to ensure ad libitum consumption.

\subsection{Sampling Procedures}

Nasopharyngeal, tracheal, lung and joint samples were collected at necropsy from 32 'case' cattle mortalities that died of BRD or were euthanized due to intractable septic arthritis and 8 'control' cattle that died of other causes. Some individual animals exhibiting infectious disease received as many as 10 antimicrobial prior to death. Cases averaged 40 days on feed (DOF) at the time of death, with a range of 15 to 71 days. Twenty of the 32 cases had gross pathological lesions consistent with mycoplasmosis, 9 were diagnosed with chronic pneumonia polyarthritis syndrome (CPPS), 5 had necrotic bronchopneumonia, and 6 cases were either chronic pneumonia or chronic bronchopneumonia. Of the eight control cattle, seven died of accidental electrocution from a singular event. These animals were near market weight. The eighth control animal was 8 DOF when it died of bloat. A total of 132 samples were collected during this study (Table 1$)$. Nasopharyngeal $(n=40)$, tracheal $(n=39)$, lung $(n=40)$ and joint $(n=13)$ tissues were obtained from case and control animals at necropsy by a field veterinarian Nasopharyngeal mucosal tissue was 
obtained by excising the nares and removing a 1-2 cm block of mucosal tissue. Deep nasal swabs were obtained using guarded nasal swabs. The trachea was transected at the mid-point between the nasal cavity and the lungs. A en bloc tracheal sample, consisting of 2-3 tracheal rings, was sectioned from the end of the transected trachea. Lung tissue, consisting of at least a $3 \times 3 \mathrm{~cm}$ sample was excised from the boundary of the healthy and diseased lung tissue, which was most commonly in the cranioventral lung lobes. Control samples were obtained from the left cranioventral lung lobe of healthy cattle. Synovial tissue $(1 \times 1 \mathrm{~cm})$ was excised from the joints of cases displaying septic arthritis. Only a single joint sample was obtained from a control animal. All sampling occurred under field conditions, with the ambient temperature frequently below $0{ }^{\circ} \mathrm{C}$. Tissues were placed in individually identified plastic specimen cups. All samples were transported in a cooler by the veterinarians collecting the samples to the veterinary clinic, where they were promptly placed in a $-20^{\circ} \mathrm{C}$ freezer. Samples were then transferred to the laboratory and stored at $-80^{\circ} \mathrm{C}$ until further processing.

Table 1. Samples used to investigate comparative microbiota from two group of animals (control vs. case (BRD) mortalities).

\begin{tabular}{ccc}
\hline Mortality & Sample Types & Sample Number \\
\hline \multirow{3}{*}{ Control mortalities $(n=8)$} & Joint & $1^{* *}$ \\
& Lung & 8 \\
& Nasopharynx & 8 \\
& Trachea & 8 \\
\hline \multirow{2}{*}{ Case (BRD) mortalities } & Joint & 12 \\
$(n=32)$ & Lung & 32 \\
& Nasopharynx & 32 \\
\hline
\end{tabular}

* Treatment: control, healthy cattle; case, cattle diagnosed with pneumonia. ${ }^{* *}$ Only one sample for joint in the control.

\subsection{DNA Extraction, Quantification and Quality Assessment}

Metagenomic DNA from samples was extracted as described by Zaheer et al. [21]. Briefly, frozen tissues $(325 \mathrm{mg}$ ) were excised in a sterile Petri dish and transferred to a sterile $2.0 \mathrm{~mL}$ safe-lock snap-cap tubes containing $0.4 \mathrm{~g}$ of sterile zirconia beads $(0.3 \mathrm{~g}$ of $0.1 \mathrm{~mm}$ and $0.1 \mathrm{~g}$ of $0.5 \mathrm{~mm})$ and $1 \mathrm{~mL}$ of resuspension buffer $(600 \mathrm{mM} \mathrm{NaCl}, 120 \mathrm{mM}$ Tris-HCl, $60 \mathrm{mM}$ EDTA, $200 \mathrm{mM}$ guanidine isothyocynate; Fisher Scientific, Ottawa, ON). Five microliters of 1:1 ratio of $\beta$-mercaptoethanol $(\beta-\mathrm{ME})$ and resuspension buffer were added to the sample tube, vortexed and then pre-heated $\left(70{ }^{\circ} \mathrm{C}\right)$. A $10 \%$ SDS $(200 \mu \mathrm{L})$ solution was added and the mixture was homogenized for 3 min on a OMNI bead Ruptor 9 (Omni International, Kennesaw, GA, USA) with setting $=5 \mathrm{M} / \mathrm{s}$. The homogenate was then incubated at $70{ }^{\circ} \mathrm{C}$ for $15 \mathrm{~min}$ in a shaking incubator at $300 \mathrm{RPM}$. The mixture was centrifuged at $4{ }^{\circ} \mathrm{C}$ for $5 \mathrm{~min}$ at $16,000 \times g$ to obtain the supernatant. To recover DNA from any remaining intact microbes, fresh resuspension buffer $(800 \mu \mathrm{L}), 1: 1$ ratio of $\beta$-ME and resuspension buffer $(5 \mu \mathrm{L})$ and $70{ }^{\circ} \mathrm{C}$ heated $10 \%$ SDS $(200 \mu \mathrm{L})$ were sequentially added to the remaining pellet, mixed, homogenized and the supernatant was collected. The supernatants were further processed for DNA extraction as described by [21].

Nasopharyngeal swabs were suspended into enzymatic buffer $(180 \mu \mathrm{L})$ containing mutanolysin $\left(300 \mathrm{U} \mathrm{mL}^{-1}\right)$ and lysozyme $\left(20 \mathrm{mg} \mathrm{mL}^{-1}\right)$, vortexed and then incubated at $37^{\circ} \mathrm{C}$ for $1 \mathrm{~h}$ in a shaking incubator at $300 \mathrm{RPM}$. Proteinase $\mathrm{K}(25 \mu \mathrm{L})$ and $200 \mu \mathrm{L}$ of AL buffer (without ethanol) from DNeasy Blood and Tissue kit (Qiagen, Toronto, ON, Canada) were then added, vortexed and incubated at $56^{\circ} \mathrm{C}$ for $30 \mathrm{~min}$. Approximately $400 \mathrm{mg}$ of zircon/silica beads $(0.3 \mathrm{~g}$ of $0.1 \mathrm{~mm}$ and $0.1 \mathrm{~g}$ of $0.5 \mathrm{~mm}$ ) were then added and mixed using a Tissue Lyser II (Qiagen) at maximum amplitude for $3 \mathrm{~min}$. The mixture was centrifuged (13,000 $\times g$ for $5 \mathrm{~min})$, and $200 \mu \mathrm{L}$ of ethanol was added to the supernatant, followed by vortexing. From this point, the DNeasy Blood and Tissue Kit was employed as per manufacturer's instructions. Subsequent to DNA isolation, quality and quantity of the 
isolated DNA was evaluated. DNA concentrations were measured by fluorescence using the Quant-iT ${ }^{\mathrm{TM}}$ PicoGreen (Thermo Fisher Scientific, Mississauga, ON, Canada). Purity of the DNA was determined by measuring the ratios of absorbance at 260/280 and 260/230 using a NanoDrop spectrophotometer (Thermo Fisher Scientific, Waltham, MA, USA). DNA preparations with a $260 / 280$ ratio between 1.7 and 2.0 and a $260 / 230$ ratio between 2.0 and 2.2 were regarded as suitable for further analysis. The extracted DNA was stored at $-80{ }^{\circ} \mathrm{C}$ until sequenced.

\section{4. $16 S$ rRNA Gene Sequencing and Analysis}

The 16S rRNA gene sequence libraries were generated using a two-step PCR protocol. The first PCR step amplified the V4 region of the 16S rRNA gene using the universal bacterial and archaeal primers 515-F (GTGCCAGCMGCCGCGGTAA) and 806-R (GGACTACHVGGGTWTCTAAT) [22]. The second PCR step was used to add a unique 10-bp barcode at the $5^{\prime}$ end of each amplicon as well as to add Illumina (Illumina, San Diego, CA, USA) adapter sequences. The $16 \mathrm{~S}$ rRNA gene amplicons were quantified using a Quant-iT PicoGreen dsDNA assay kit (Invitrogen, Burlington, ON, Canada), pooled in equimolar ratios, and then purified with AMPure XP beads (Beckman Coulter, Mississauga, ON, Canada). Sequencing of $16 \mathrm{~S}$ rRNA gene amplicons was carried out using an Illumina MiSeq ( $2 \times 250$; San Diego, CA, USA) and the MiSeq Reagent Kit v2 (500 cycles; Illumina) according to manufacturer's instructions. All PCR amplification and sequencing steps were carried out at Genome Quebec (Montreal, QC, Canada).

The 16S rRNA gene sequences were processed using QIIME2 [23] and the R-package DADA2 (Version 1.40) denoise method. Briefly, the forward and reverse reads were each truncated at a length of $240 \mathrm{bp}$, quality control was performed for the reads using the QIME2, with chimeric sequences identified and removed. The reads were merged and taxonomy assigned so as to generate operational taxonomic units (OTUs) at 97\% similarity, using the naïve Bayesian RDP classifier [24] and the Greengenes reference database [25]. The number of OTUs per sample and the Shannon diversity index were calculated in R using Phyloseq 1.20.0 [26] and vegan 2.4.4 [27] was used to determine the Bray-Curtis dissimilarities. Differential bacterial taxonomy of cattle that succumbed to BRD as compared to controls were identified using R-package Deseq2 [28] with Fold Change. Sequences have been submitted to NCBI under Bioproject PRJNA788973.

\subsection{Statistical Analysis}

Nasopharynx, tracheas, lung and joint samples were randomly subsampled to 16,000 sequences, prior to the calculation of the diversity metrics and Bray-Curtis dissimilarities. Only OTUs with at least 50 reads in the samples were included in the analysis. The number of OTUs and Shannon diversity index were analyzed in R v.3.6.1 by mortality using a linear mixed model implemented with the lmer function in lme4 $\mathrm{v} 1.1 .15$ package [29]. The linear mixed model included the random effect of the sample site and fixed effects of BRD case or control mortalities. The number of OTUs and Shannon diversity index from BRD mortalities were analyzed in $R$ v. 3.6.1 by tissue type using a linear mixed model implemented with the lmer function in lme4 v. 1.1.15 package [28]. The linear mixed model included the random effect of the sample site and fixed effect of tissue type. Post hoc comparisons were performed within each sample type using Tukey's honestly significant difference test by postHoc package v.0.1.3. Microbial community structure of nasopharynx, trachea and lung samples were analyzed with vegan using permutational multivariate analysis of variance (PERMANOVA; Adonis function; 10,000 permutations) of the Bray-Curtis dissimilarities to assess the difference between BRD case and control mortalities. Similarly, microbial communities of nasopharyngeal, tracheal, lung and joint samples from BRD mortalities were analyzed with vegan using permutational multivariate analysis of variance (PERMANOVA; Adonis function; 10,000 permutations) of the BrayCurtis dissimilarities. The differential taxonomy based on genus level was analyzed using GMPR to adjust the data, hierarchical cluster, clustering distance with Euclidean and Log 
fold changes for comparison. Joint sample data were not subjected to statistical analysis between BRD and control mortalities due to only having a single control sample.

\section{Results}

\section{1. $16 S$ rRNA Sequence Data}

A total of 132 samples (Table 1) were used to assess microbiomes and sequences were filtered for size, quality, and for the presence of chimeras. A total of 7,768,164 reads (median $=59,109$, minimum $=4404$, and maximum $=115,795)$ were used to identify 4661 unique bacterial operational taxonomic units (OTUs).

A decreasing trend in alpha diversity was observed for BRD cases as compared to controls as identified by both the number of OTUs per sample (richness) and the Shannon diversity index in all tissue types (Figure 1). A lower $(p<0.05)$ number of OTUs per sample (richness) and the Shannon diversity index were detected for both tracheal and lung samples, while there was no difference $(p>0.05)$ in the number of OTUs in nasopharyngeal samples from cases vs. controls. No statistical analysis could be conducted for the joint samples due to the availability of a single control sample (Figure 1a). Both the number of OTUs per sample and the Shannon index had similar trends among all samples (Figure 1b). The number of OTUs per sample (richness) and the Shannon index for joint samples was similar to trachea while this estimate was lower $(p<0.05)$ for the nasopharynx and higher $(p<0.05)$ for lung samples (Figure 1b).

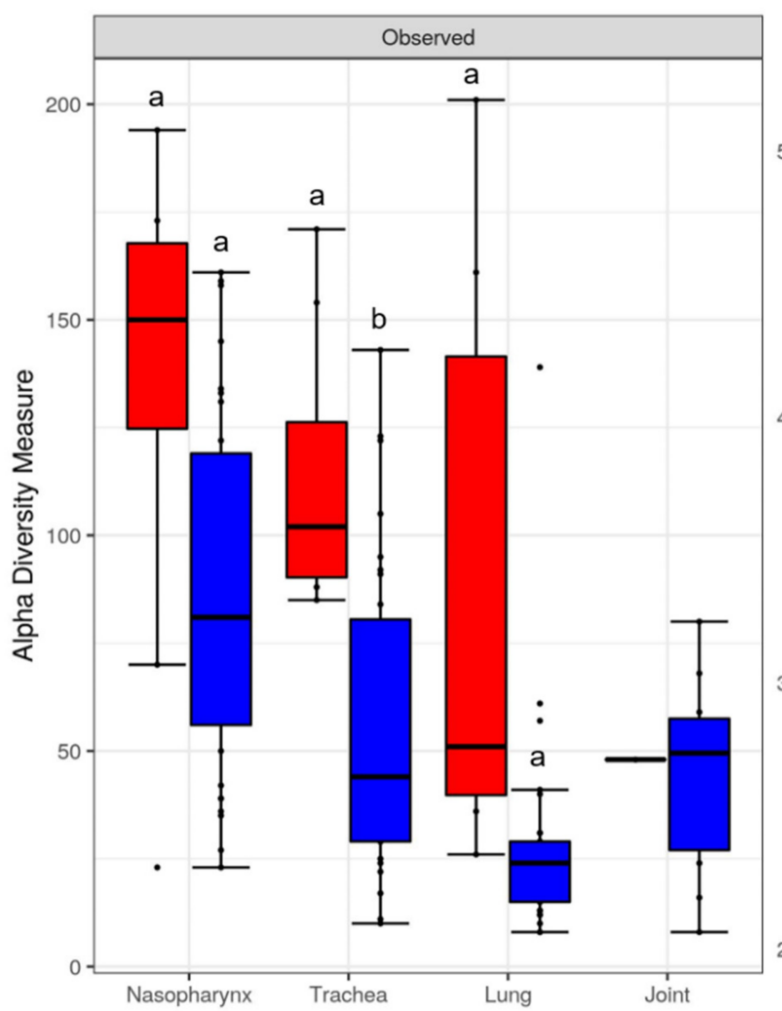

Nasopharynx

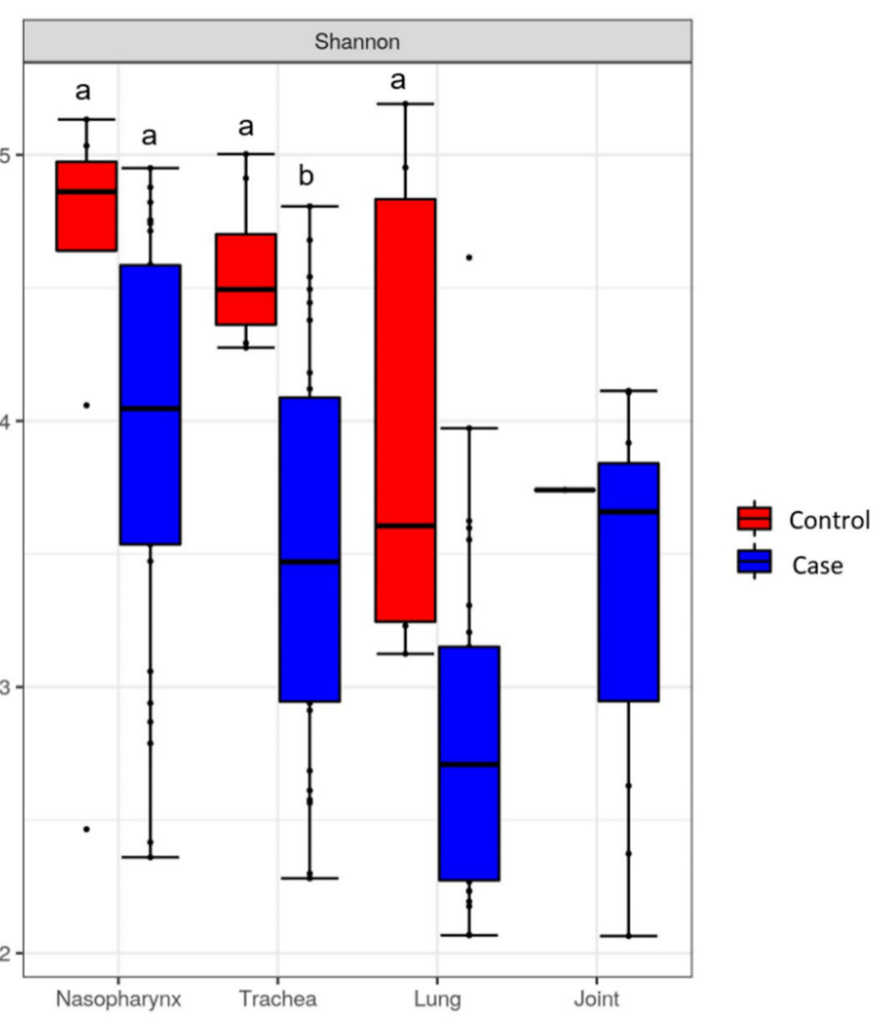

(a)

Figure 1. Cont. 

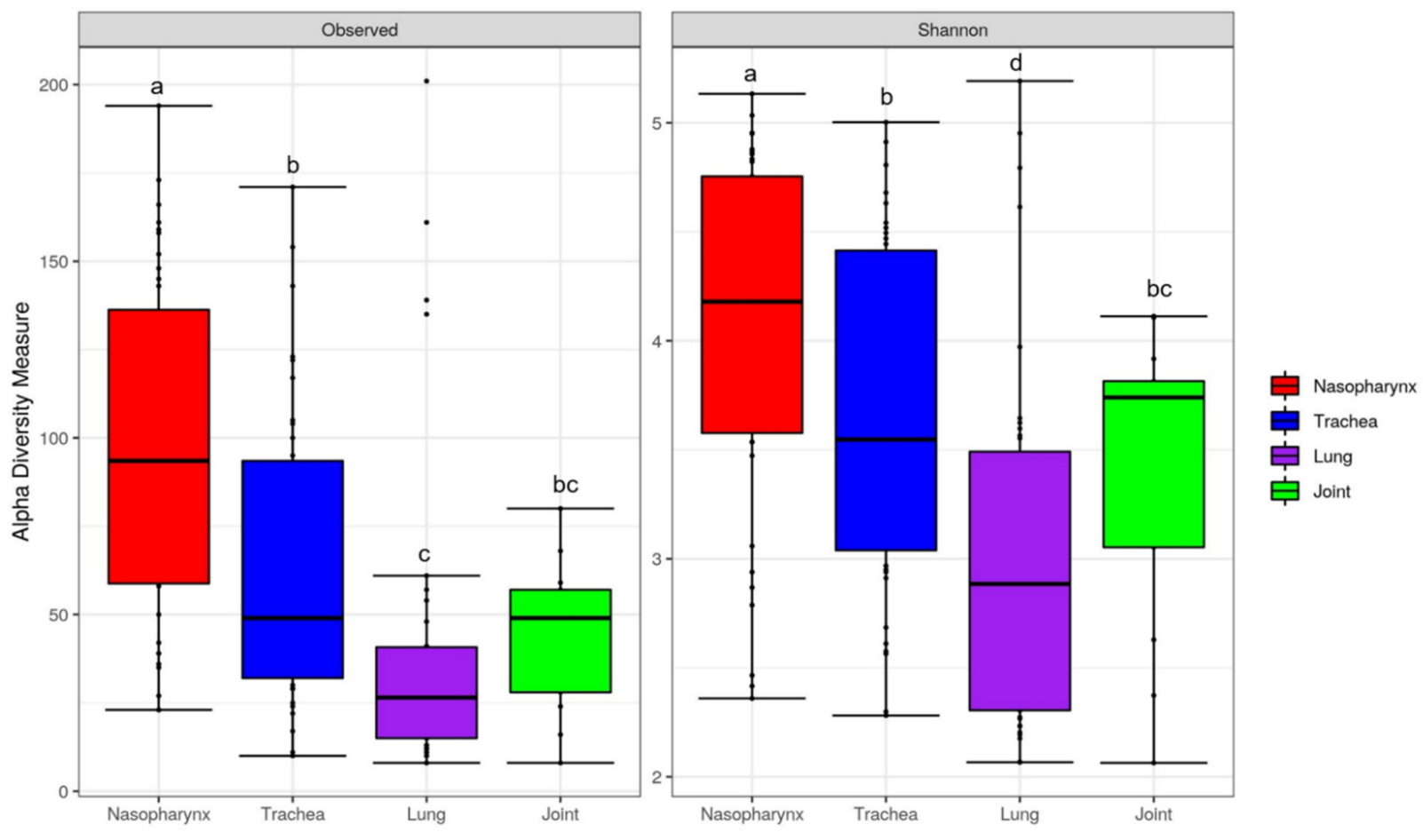

(b)

Figure 1. (a) Comparative alpha-diversity (observed and Shannon) of OTUs in respiratory tract and joint microbiota from case and control cattle. Different lowercase letters associated with each sample type differ at $p<0.05$. (b) Comparative alpha-diversity (observed and Shannon) of OTUs in respiratory tract and joint microbiota from BRD mortalities (cases). Different lowercase letters differ at $p<0.05$.

\subsection{Comparative Tissue Microbiota of Cases and Controls}

Permutational multivariate analysis of variance (PERMANOVA) indicated that cause of death was associated with the microbial community structure of the nasopharynx $\left(R^{2}=0.042 ; p=0.003\right)$, trachea $\left(R^{2}=0.048 ; p=0.005\right)$ and lungs $\left(R^{2}=0.063 ; p=0.005\right)$ but not the joints. Overall, the microbiota from all three locations of the respiratory tract (nasopharynx, trachea and lung) were dissimilar between cases and controls. Similarly, PERMANOVA revealed that tissue types from cases differed in their microbial community structure between the nasopharynx and lung $\left(R^{2}=0.051 ; p=0.0001\right)$, trachea and lung $\left(R^{2}=0.044 ; p=0.0001\right)$, joint and nasopharynx $\left(R^{2}=0.032 ; p=0.05\right)$ as well as the joint and lung $\left(\mathrm{R}^{2}=0.047 ; p=0.02\right)$.

Twenty-nine different bacterial phyla were observed among all samples, but only Proteobacteria, Tenericutes, Bacteroidetes, Firmicutes, Actinobacteria, Fusobacteria and Euryarchaeota had a relative abundance greater than $1.0 \%$ (Figure 2). Five phyla represented $94.1 \%$ of the total bacterial community in both cases and controls: Proteobacteria $(39.4 \%)$, Tenericutes (17.3\%) Bacteroidetes (17.2\%), Firmicutes (15.6\%) and Actinobacteria (4.6\%). The relative abundance of these phyla differed between cases and controls depending on the type of tissues. A predominance of Proteobacteria was noted in the nasopharynx (43.6\%), trachea $(45.8 \%)$, lung $(45.4 \%)$ and joint $(37.7 \%)$ of cases as compared to samples from controls, $14.9 \%, 26.3 \%, 22.7 \%$ and $27.4 \%$, respectively. Tenericutes, of which Mycoplasma is a genus, were prevalent in all tissue types (Figure 2), comprising 20.5\% of bacteria in joints as compared to $8.9 \%$ in nasopharynx, $22.7 \%$ in trachea and $36.0 \%$ in lungs. 


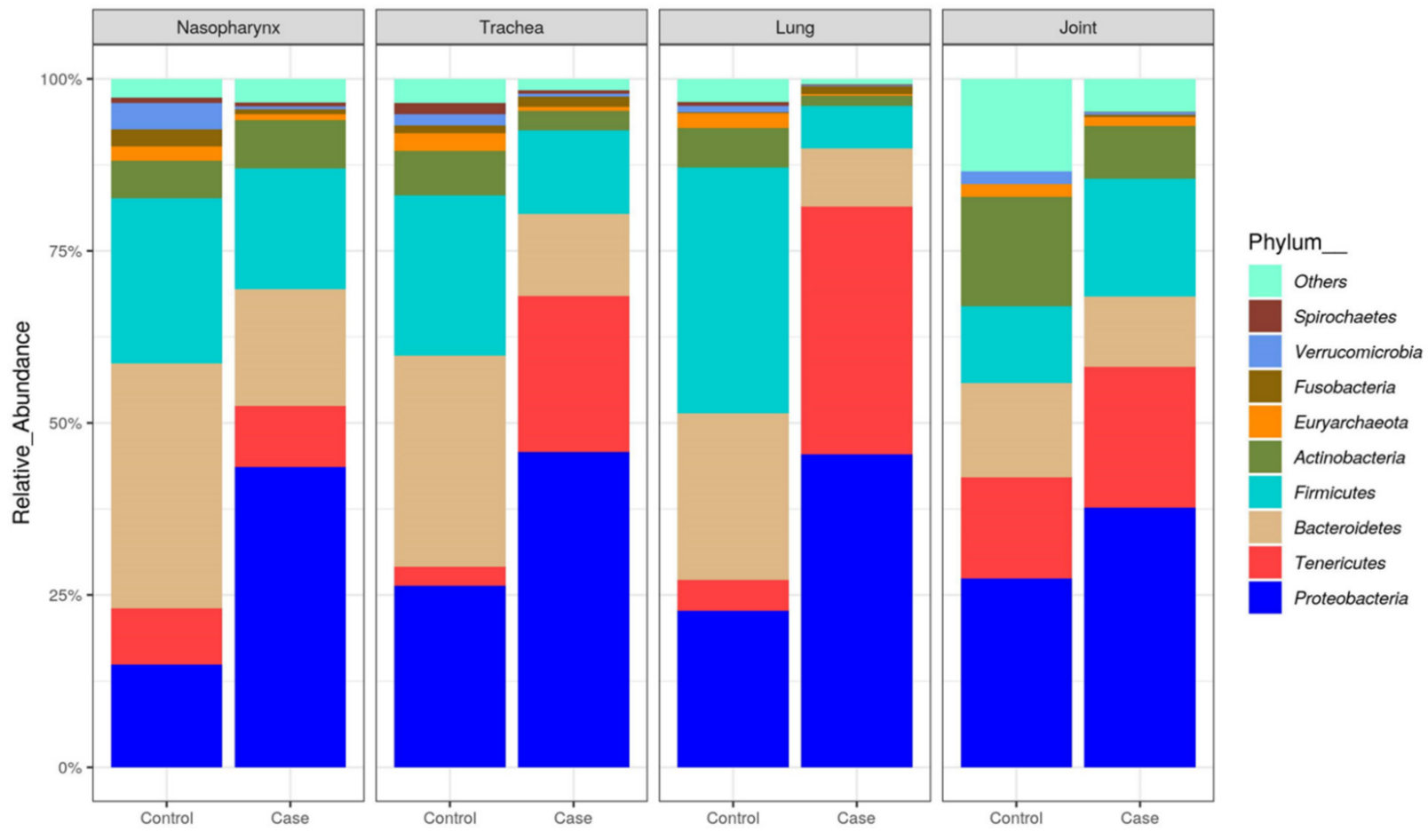

Figure 2. Relative abundance of bacterial 16S rRNA gene sequences at the phylum level observed in the nasopharynx, trachea and lung of cattle succumbed to BRD (cases) as compared to controls. All other classified OTUs comprising less than $1 \%$ of the total abundance are represented as others/unassigned taxa.

Both Mycoplasma and Psychrobacter were the predominant genera in samples, with a relative abundance of $17.1 \%$ and $19.9 \%$, respectively, followed by Prevotella 1 (9.2\%), Mannheimia (8.8\%), Histophilus (4.0\%), Clostridium (3.0\%), Corynebacterium (1.36\%), Bacteroides $(1.3 \%)$ and Shigella $(0.9 \%)$ in both cases and controls (Figure 3$)$. Compared to controls, the relative abundance of Mycoplasma and Psychrobacter increased, while Prevotella decreased in all sample types associated with cases (Figure 3). Bacterial genera associated with cases also differed among sample types (Figure 3). For example, Mycoplasma was more abundant in joint, lung, trachea and nasopharynx at 20.5\%, 36.0\%, 22.4\% and $8.8 \%$, respectively. The relative abundance of both Mannheimia and Histophilus was 2.0 and $0.9 \%$ in joints as compared to the nasopharynx (5.7 and 2.6\%), trachea (2.5 and 7.7\%) and lung (21.8 and $10.4 \%$ ). In addition, the relative abundance of Prevotella in joints was $4.1 \%$ as compared to nasopharynx $(8.6 \%)$, trachea $(6.2 \%)$ and lungs (3.6\%). The relative abundance of Shigella associated with joints was $3.2 \%$ as compared to nasopharynx $(0.5 \%)$, trachea $(2.7 \%)$ and lungs (3.8\%). 

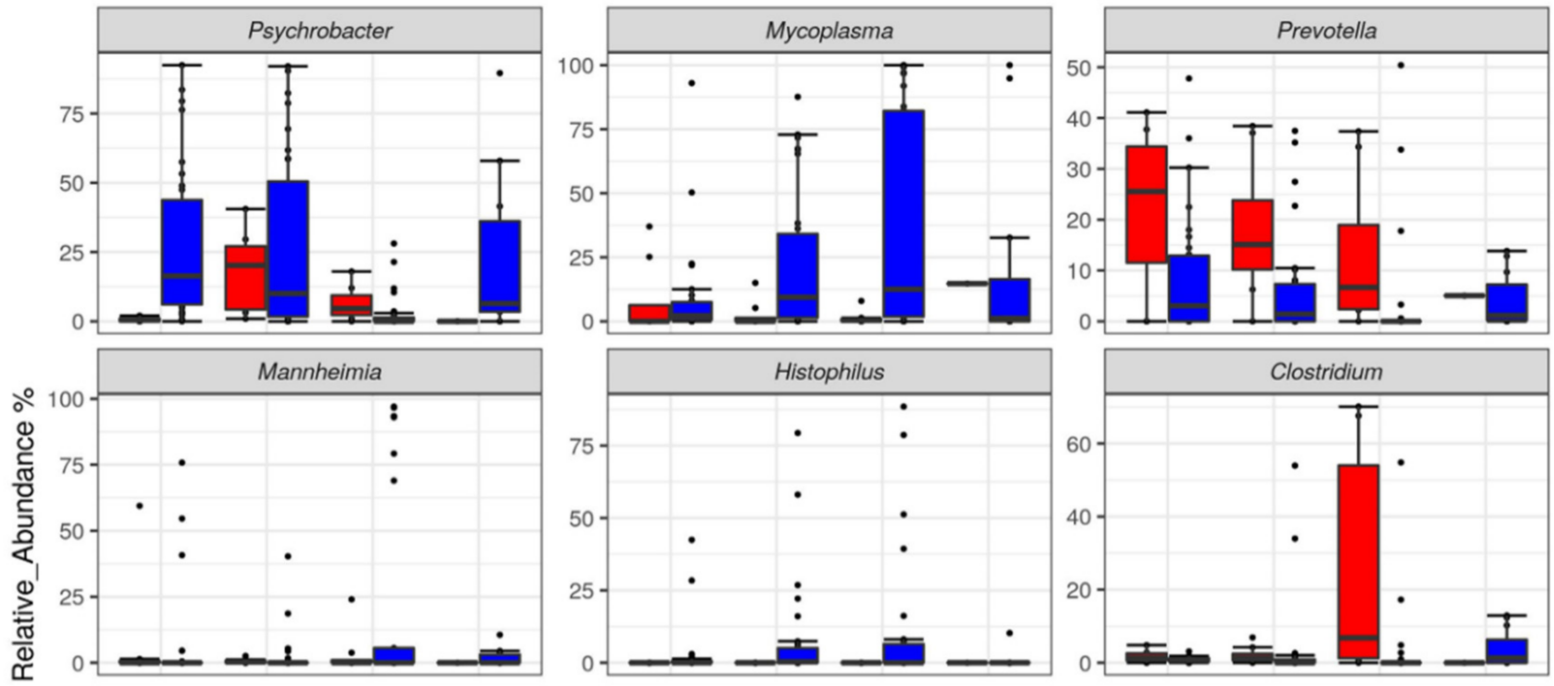

Control

Case
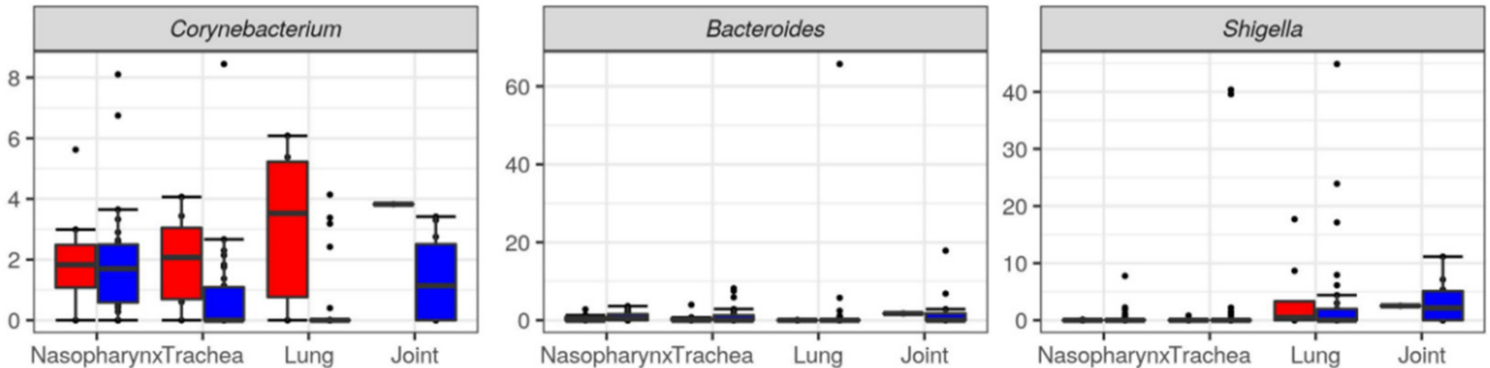

Figure 3. Relative abundance of top nine bacterial genera in tissue from 'cases' and 'controls'. Black dots indicate outlier data points.

Differential taxonomic comparison at the genus level indicated that the bacteria linked to cases (Figure 4), including Mycoplasma associated with lungs, and Pasteurella associated with the trachea and lungs were higher than controls. Histophilus and Trueperella were higher $(p<0.001)$ in all three types of tissues from the respiratory tract of BRD cases as compared to controls. In addition, Psychrobacter in nasopharynx and Bacteroides in lungs was $(p<0.001)$ higher in cattle that died of BRD as compared to controls. 


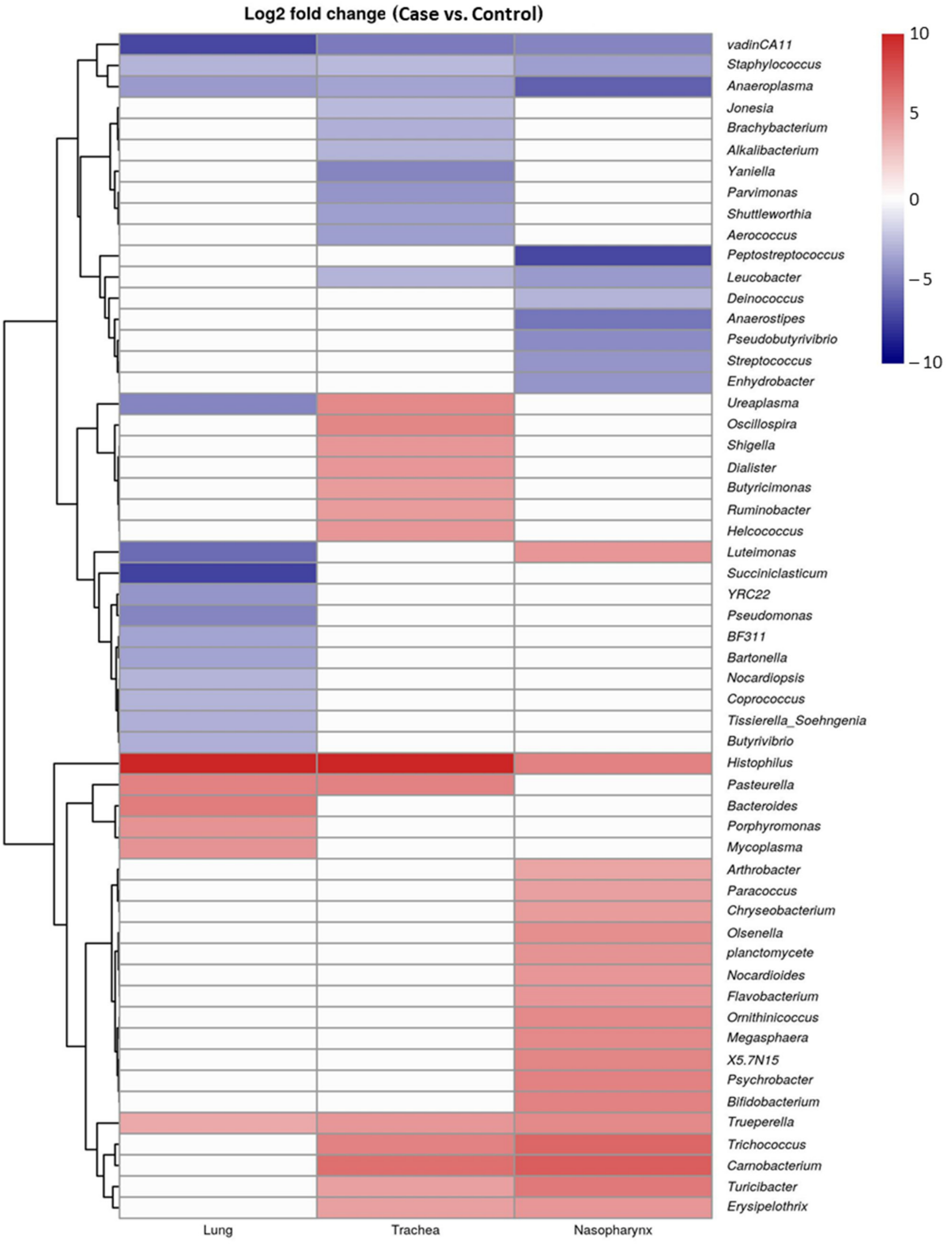

Figure 4. Heatmap of mean relative abundance of bacterial genera identified in respiratory tract samples from BRD cases and controls. 


\section{Discussion}

This is the first study to characterize the microbiome of the respiratory tract and joints of feedlot cattle afflicted with arthritis and/or pneumonia. Previous microbiome studies of feedlot cattle have been limited to samples from the nasopharyngeal passages of healthy and sick animals, or the upper and lower respiratory tracts of healthy cattle $[5,7,8,16,18]$, whereas the current study utilized tissue samples obtained at the time of postmortem examination from cattle that had either been euthanized or died naturally of nonresponsive arthritis or pneumonia. It is salient that the calves averaged 40 days on feed (DOF) at the time of death, and that all received metaphylaxis on arrival, with those displaying clinical disease being treated with antimicrobials at least once prior to death. The timing of the deaths (DOF) coupled with the animals being refractory to multiple courses of antimicrobials is highly suggestive of mycoplasmosis. In fact, five animals had overt lung lesions consistent with mycoplasmosis, while another nine animals were diagnosed with chronic pneumonia and polyarthritis syndrome (CPPS), pathognomonic for M. bovis. Thus, the cases in this study are representative of calves that acquired bovine respiratory disease (BRD) within days to weeks of arrival at the feedlot, with most possessing lesions that were consistent with mycoplasmosis.

As has been previously reported, the alpha-diversity index was consistently lower for the microbiota of cases versus the controls across all tissues. The alpha-diversity in the nasopharynx of cases and controls was consistent with a previous study [30], wherein the alpha-diversity in the nasopharynx did not differ between calves diagnosed with $\mathrm{BRD}$ and healthy calves, regardless of age. The lower alpha-diversity in the trachea was also consistent with a study that examined steers with BRD as compared to healthy penmates [16]. Cases in our study also had a lower alpha-diversity of lung microbiota as compared to controls. Furthermore, cases exhibited a lower alpha-diversity in the joint and lung tissues as compared to the nasopharynx. As a diverse microbiota could provide protection against infections, a loss of diversity and shifts in the microbiota composition may establish a niche that enables virulent members of the BRD bacterial complex to proliferate and establish infections.

Previous culture-independent approaches have found differences in the nasopharyngeal and tracheal bacterial communities of healthy cattle and those diagnosed with BRD $[5,16,31]$. The current study detected significant differences in the composition of the microbiota in the nasopharynx as well as the tracheas and lungs of cattle that succumbed to BRD as compared to those that died of other non-pathogenic causes. The structure of bacterial communities of both the upper and lower respiratory tract were altered as a result of BRD. Our results also showed significant differences in the microbiota of the nasopharynx, trachea, lung and joint within BRD mortalities. Others also found distinct microbial communities between the nasopharynx and trachea of post-weaned Piedmontese calves or steers with or without BRD $[16,17]$. The fact that tracheal microbiota partly share common bacterial community members with the nasopharyngeal microbiota or lung microbiota is not surprising, as these sites are anatomically continuous.

In agreement with our study, Proteobacteria, Tenericutes, Bacteroidetes, Firmicutes and Actinobacteria have been reported to be the most abundant phyla in nasopharynx and trachea of recently weaned calves and feedlot cattle [8,17-19,32]. The second most abundant phylum was Tenericutes (17.3\%), which contains the class Mollicutes that includes Mycoplasma. Tenericutes have been reported to be the most abundant phylum in the nasopharynx and trachea of feedlot cattle comprising more than $40 \%$ of the total bacterial community [16,32]. The current study revealed a predominance of Proteobacteria in nasopharynx, trachea and lung, and Tenericutes in trachea and lungs of cattle that died of BRD as compared to controls. Furthermore, there was a predominance of Tenericutes in the trachea, lungs and joints, with these members being lower in the nasopharynx of cattle that died of BRD. 
The most abundant genera detected in the nasopharynx, trachea and lung of cases and controls were Mycoplasama, Psychrobacter, Mannheimia, Prevotella and Histophilus. This finding is similar to that observed in the nasopharynx and trachea of feedlot steers diagnosed with or without pneumonia [16]. The dominance of Mycoplasma in the respiratory tract of both cases and controls is in agreement with a recent study [17], which found that Mycoplasma represented $27.3 \%$ and $76.7 \%$ of the genera in nasopharynx and trans-tracheal samples, respectively. A greater relative abundance of Mycoplasma in the tracheas and lungs of cattle was not unexpected since most of the BRD cattle were euthanized because they were presumptively deemed chronic mycoplasmosis cases. Similar results were reported in tracheal samples [16] with more $M$. bovis in feedlot cattle diagnosed with bronchopneumonia or in bronchoalveolar lavage samples from necropsied feedlot cattle diagnosed with chronic suppurative pneumonia [11,14,31].

A number of Mycoplasma species including M. arginini, M. bovirhinis, M. bovis, and M. dispar have been associated with BRD in cattle, with $M$. bovis being the most abundant [31]. Furthermore, higher Mycoplasma abundance both in joints and lungs of cases in the present study are supportive of mycoplasmas being a causative agent of BRD and septic arthritis [15,33], which when found concurrently in the same animal is known as chronic pneumonia polyarthritis syndrome (CPPS). In an outbreak of unresponsive arthritis and pneumonia as a result of $M$. bovis infection in young calves in Jordan, lesions mediated by $M$. bovis were found in both the lungs and joints [34]. Similar findings were reported in another study where the pulmonary lesions were accompanied by polyarthritis [35].

The other most abundant genus was Psychrobacter, a member of the phylum Proteobacteria. This genus was found in the nasopharynx, trachea, lung and joint of both cases and controls. Others have also associated Psychrobacter with these tissues $[5,16,19,30]$, but at low abundance and its presence in the upper respiratory tract has been inconsistent [7], with its relative abundances not differing between cases and controls. In the current study, Psychrobacter appeared to be enriched in the nasopharynx of cattle with pneumonia (27.4\% versus $0.6 \%$ of controls). Psychrobacters are characteristically halotolerant and psychrophilic and have been isolated from diverse habitats including brain tissue, eye, urethra, blood and cerebrospinal fluid from humans [36], but their clinical significance, if any, is largely unknown.

Although Mannheimia, Mycoplasma, Histophilus and Pasteurella are the most well-known bacterial pathogens associated with BRD in feedlot cattle [5,10,12,37-39], the results did not show an increase in relative abundance of Mannheimia between cases and controls. $M$. haemolytica is a commensal within the upper respiratory tract of cattle, with serotype 2 strains being less virulent in cattle than serotype 1 and 6 strains [40], a trait that cannot be distinguished via 16SrRNA sequencing. Others have found that the relative abundance of Mannheimia was similar in cattle that developed BRD as compared to their healthy counterparts [20]. Higher occurrence of Pasteurella in trachea and lung samples, and Histophilus in nasopharynx, trachea and lung samples from cattle with BRD as compared to controls likely reflects the important role that these genera play in this infectious disease.

A few genera that are typically associated with the gut were also found in the respiratory tract of cattle. Bacteroides were found in the tracheas and lungs and were more abundant in cattle that died of BRD than in control mortalities. Prevotella was also found in all samples, but at similar levels of abundance between cases and controls. Others reported that the relative abundance of these genera in the nasopharynx was similar among healthy and pneumonic dairy calves [30]. More Bacteroides in both nasopharynx and tracheal samples from BRD feedlot cattle have been noted as compared to healthy cattle [16]. Although the potential role of these genera in the respiratory tract of cattle remains unknown, the predominance of these genera in the respiratory tract might reflect microbial seeding of this organ as a result of regurgitation of feedstuffs during rumination, eructation, or at the time of death. 
In conclusion, distinct bacterial communities inhabit the respiratory tract and joints of control cattle mortalities versus the same anatomical locations of those that died of BRD or arthritis. Cases harbored less diverse respiratory tract microbiota than controls, with a higher relative abundance of BRD bacterial pathogens. Similarly, bacterial communities in the joints and lungs of cattle that died of BRD were less diverse in the nasopharynx, with a higher relative abundance of bacterial pathogens in the joints or lungs than in the nasopharynx and trachea.

Author Contributions: Conceptualization, T.A.M. and M.J.; Methodology, C.L. and R.Z.; Formal Analysis, C.L. and R.Z.; Investigation, C.L., R.Z. and A.K.; Data Curation, C.L. and R.Z.; WritingOriginal Draft Preparation, C.L. and R.Z.; Writing-Review and Editing, C.L., R.Z., M.J. and T.A.M.; Funding Acquisition, T.A.M. and M.J. All authors have read and agreed to the published version of the manuscript.

Funding: This research was funded by the Saskatchewan Agricultural Development Fund (Grants \#20060116, \#20130170, \#20160253) and the Beef Cattle Research Council (Grant ANH.30.17).

Institutional Review Board Statement: Not applicable.

Informed Consent Statement: Not applicable.

Data Availability Statement: All Illumina sequence read data from current study have been deposited to the NCBI database as Short Read Archive (SRA) under BioProject ID: PRJNA788973.

Acknowledgments: The authors thank the field veterinarians at Veterinary Agri-Health Services (Airdrie, Alberta) and the feedlot producers that were willing to make the collected samples available to the research team.

Conflicts of Interest: The authors declare no conflict of interest. The funders had no role in the design of the study; in the collection, analyses, or interpretation of data; in the writing of the manuscript, or in the decision to publish the results.

\section{References}

1. Griffin, D. Economic Impact Associated with Respiratory Disease in Beef Cattle. Vet. Clin. N. Am. Food Anim. Pract. 1997, 13, 367-377. [CrossRef]

2. Cernicchiaro, N.; White, B.J.; Renter, D.G.; Babcock, A.H. Evaluation of Economic and Performance Outcomes Associated with the Number of Treatments after an Initial Diagnosis of Bovine Respiratory Disease in Commercial Feeder Cattle. Am. J. Vet. Res. 2013, 74, 300-309. [CrossRef] [PubMed]

3. Johnson, K.K.; Pendell, D.L. Market Impacts of Reducing the Prevalence of Bovine Respiratory Disease in United States Beef Cattle Feedlots. Front. Vet. Sci. 2017, 4, 189. [CrossRef]

4. Aebi, M.; Van den Borne, B.H.; Raemy, A.; Steiner, A.; Pilo, P.; Bodmer, M. Mycoplasma Bovis Infections in Swiss Dairy Cattle: A Clinical Investigation. Acta Vet. Scand. 2015, 57, 10. [CrossRef]

5. Holman, D.B.; McAllister, T.A.; Topp, E.; Wright, A.-D.G.; Alexander, T.W. The Nasopharyngeal Microbiota of Feedlot Cattle That Develop Bovine Respiratory Disease. Vet. Microbiol. 2015, 180, 90-95. [CrossRef] [PubMed]

6. Confer, A.W. Update on Bacterial Pathogenesis in BRD. Anim. Health Res. Rev. 2009, 10, 145-148. [CrossRef]

7. Holman, D.B.; Timsit, E.; Alexander, T.W. The Nasopharyngeal Microbiota of Feedlot Cattle. Sci. Rep. 2015, 5, 15557. [CrossRef] [PubMed]

8. Holman, D.B.; Timsit, E.; Amat, S.; Abbott, D.W.; Buret, A.G.; Alexander, T.W. The Nasopharyngeal Microbiota of Beef Cattle before and after Transport to a Feedlot. BMC Microbiol. 2017, 17, 70. [CrossRef]

9. Hodgson, P.D.; Aich, P.; Manuja, A.; Hokamp, K.; Roche, F.M.; Brinkman, F.S.L.; Potter, A.; Babiuk, L.A.; Griebel, P.J. Effect of Stress on Viral-Bacterial Synergy in Bovine Respiratory Disease: Novel Mechanisms to Regulate Inflammation. Comp. Funct. Genom. 2005, 6, 244-250. [CrossRef]

10. Rice, J.A.; Carrasco-Medina, L.; Hodgins, D.C.; Shewen, P.E. Mannheimia Haemolytica and Bovine Respiratory Disease. Anim. Health Res. Rev. 2007, 8, 117-128. [CrossRef]

11. Caswell, J.L. Failure of Respiratory Defenses in the Pathogenesis of Bacterial Pneumonia of Cattle. Vet. Pathol. 2014, 51, 393-409. [CrossRef] [PubMed]

12. Booker, C.W.; Abutarbush, S.M.; Morley, P.S.; Jim, G.K.; Pittman, T.J.; Schunicht, O.C.; Perrett, T.; Wildman, B.K.; Fenton, R.K.; Guichon, P.T. Microbiological and Histopathological Findings in Cases of Fatal Bovine Respiratory Disease of Feedlot Cattle in Western Canada. Can. Vet. J. 2008, 49, 473. [PubMed]

13. Dungworth, D.; Tyler, W.; Plopper, C. Morphological Methods for Gross and Microscopic Pathology. In Toxicology of Inhaled Materials; Springer: Berlin/Heidelberg, Germany, 1985; pp. 229-258. 
14. Gagea, M.I.; Bateman, K.G.; Van Dreumel, T.; McEwen, B.J.; Carman, S.; Archambault, M.; Shanahan, R.A.; Caswell, J.L. Diseases and Pathogens Associated with Mortality in Ontario Beef Feedlots. J. Vet. Diagn. Investig. 2006, 18, 18-28. [CrossRef] [PubMed]

15. Maunsell, F.P.; Donovan, G.A. Mycoplasma Bovis Infections in Young Calves. Vet. Clin. N. Am. Food Anim. Pract. 2009, 25, 139-177. [CrossRef] [PubMed]

16. Timsit, E.; Workentine, M.; Van der Meer, F.; Alexander, T. Distinct Bacterial Metacommunities Inhabit the Upper and Lower Respiratory Tracts of Healthy Feedlot Cattle and Those Diagnosed with Bronchopneumonia. Vet. Microbiol. 2018, 221, 105-113. [CrossRef]

17. Nicola, I.; Cerutti, F.; Grego, E.; Bertone, I.; Gianella, P.; D’Angelo, A.; Peletto, S.; Bellino, C. Characterization of the Upper and Lower Respiratory Tract Microbiota in Piedmontese Calves. Microbiome 2017, 5, 152. [CrossRef]

18. Timsit, E.; Workentine, M.; Schryvers, A.B.; Holman, D.B.; Van der Meer, F.; Alexander, T.W. Evolution of the Nasopharyngeal Microbiota of Beef Cattle from Weaning to 40 Days after Arrival at a Feedlot. Vet. Microbiol. 2016, 187, 75-81. [CrossRef]

19. Gaeta, N.C.; Lima, S.F.; Teixeira, A.G.; Ganda, E.K.; Oikonomou, G.; Gregory, L.; Bicalho, R.C. Deciphering Upper Respiratory Tract Microbiota Complexity in Healthy Calves and Calves That Develop Respiratory Disease Using Shotgun Metagenomics. J. Dairy Sci. 2017, 100, 1445-1458. [CrossRef]

20. Zeineldin, M.; Lowe, J.; De Godoy, M.; Maradiaga, N.; Ramirez, C.; Ghanem, M.; Abd El-Raof, Y.; Aldridge, B. Disparity in the Nasopharyngeal Microbiota between Healthy Cattle on Feed, at Entry Processing and with Respiratory Disease. Vet. Microbiol. 2017, 208, 30-37. [CrossRef]

21. Zaheer, R.; Noyes, N.; Ortega Polo, R.; Cook, S.R.; Marinier, E.; Van Domselaar, G.; Belk, K.E.; Morley, P.S.; McAllister, T.A. Impact of Sequencing Depth on the Characterization of the Microbiome and Resistome. Sci. Rep. 2018, 8, 5890. [CrossRef]

22. Walters, W.; Hyde, E.R.; Berg-Lyons, D.; Ackermann, G.; Humphrey, G.; Parada, A.; Gilbert, J.A.; Jansson, J.K.; Caporaso, J.G.; Fuhrman, J.A.; et al. Improved Bacterial 16S RRNA Gene (V4 and V4-5) and Fungal Internal Transcribed Spacer Marker Gene Primers for Microbial Community Surveys. mSystems 2016, 1, e00009-15. [CrossRef]

23. Bolyen, E.; Rideout, J.R.; Dillon, M.R.; Bokulich, N.A.; Abnet, C.C.; Al-Ghalith, G.A.; Alexander, H.; Alm, E.J.; Arumugam, M.; Asnicar, F. Reproducible, Interactive, Scalable and Extensible Microbiome Data Science Using QIIME 2. Nat. Biotechnol. 2019, 37, 852-857. [CrossRef] [PubMed]

24. Wang, Q.; Garrity, G.M.; Tiedje, J.M.; Cole, J.R. Naïve Bayesian Classifier for Rapid Assignment of RRNA Sequences into the New Bacterial Taxonomy. Appl. Environ. Microbiol. 2007, 73, 5261-5267. [CrossRef]

25. McDonald, D.; Price, M.N.; Goodrich, J.; Nawrocki, E.P.; DeSantis, T.Z.; Probst, A.; Andersen, G.L.; Knight, R.; Hugenholtz, P. An Improved Greengenes Taxonomy with Explicit Ranks for Ecological and Evolutionary Analyses of Bacteria and Archaea. ISME J. 2012, 6, 610-618. [CrossRef] [PubMed]

26. McMurdie, P.J.; Holmes, S. Phyloseq: An R Package for Reproducible Interactive Analysis and Graphics of Microbiome Census Data. PLoS ONE 2013, 8, e61217. [CrossRef]

27. Oksanen, J.; Blanchet, F.G.; Kindt, R.; Legendre, P.; Minchin, P.R.; O'hara, R.; Simpson, G.L.; Solymos, P.; Stevens, M.H.H.; Wagner, H. Vegan: Community Ecology Package. R Package Version 2.2-1. 2015. Available online: http://cran.rproject.org/ package= vegan (accessed on 14 December 2021).

28. Love, M.I.; Huber, W.; Anders, S. Moderated Estimation of Fold Change and Dispersion for RNA-Seq Data with DESeq2. Genome Biol. 2014, 15, 550. [CrossRef]

29. Bates, D.; Mächler, M.; Bolker, B.; Walker, S. Fitting Linear Mixed-Effects Models Using Lme4. J. Stat. Softw. 2015, 67, 1-48. [CrossRef]

30. Lima, S.F.; Teixeira, A.G.V.; Higgins, C.H.; Lima, F.S.; Bicalho, R.C. The Upper Respiratory Tract Microbiome and Its Potential Role in Bovine Respiratory Disease and Otitis Media. Sci. Rep. 2016, 6, 29050. [CrossRef]

31. Klima, C.L.; Holman, D.B.; Ralston, B.J.; Stanford, K.; Zaheer, R.; Alexander, T.W.; McAllister, T.A. Lower Respiratory Tract Microbiome and Resistome of Bovine Respiratory Disease Mortalities. Microb. Ecol. 2019, 78, 446-456. [CrossRef]

32. Stroebel, C.; Alexander, T.; Workentine, M.L.; Timsit, E. Effects of Transportation to and Co-Mingling at an Auction Market on Nasopharyngeal and Tracheal Bacterial Communities of Recently Weaned Beef Cattle. Vet. Microbiol. 2018, 223, 126-133. [CrossRef]

33. Krysak, D.E. Chronic Pneumonia and Polyarthritis Syndrome in a Feedlot Calf. Can. Vet. J. 2006, 47, 1019. [PubMed]

34. Hananeh, W.M.; Al Momani, W.M.; Ababneh, M.M.; Abutarbush, S.M. Mycoplasma Bovis Arthritis and Pneumonia in Calves in Jordan: An Emerging Disease. Vet. World 2018, 11, 1663-1668. [CrossRef] [PubMed]

35. Constant, C.; Nichols, S.; Desrochers, A.; Babkine, M.; Fecteau, G.; Lardé, H.; Fairbrother, J.-H.; Francoz, D. Clinical Findings and Diagnostic Test Results for Calves with Septic Arthritis: 64 Cases (2009-2014). J. Am. Vet. Med. Assoc. 2018, 252, 995-1005. [CrossRef] [PubMed]

36. Hudson, M.J.; Hollis, D.G.; Weaver, R.E.; Galvis, C.G. Relationship of CDC group EO-2 and Psychrobacter immobilis. J. Clin. Microbiol. 1987, 25, 1907-1910. [CrossRef]

37. Angen, Ø.; Thomsen, J.; Larsen, L.E.; Larsen, J.; Kokotovic, B.; Heegaard, P.M.H.; Enemark, J.M.D. Respiratory Disease in Calves: Microbiological Investigations on Trans-Tracheally Aspirated Bronchoalveolar Fluid and Acute Phase Protein Response. Vet. Microbiol. 2009, 137, 165-171. [CrossRef] [PubMed]

38. Griffin, D.; Chengappa, M.M.; Kuszak, J.; McVey, D.S. Bacterial Pathogens of the Bovine Respiratory Disease Complex. Vet. Clin. N. Am. Food Anim. Pract. 2010, 26, 381-394. [CrossRef] [PubMed] 
39. Francoz, D.; Buczinski, S.; Bélanger, A.M.; Forté, G.; Labrecque, O.; Tremblay, D.; Wellemans, V.; Dubuc, J. Respiratory Pathogens in Québec Dairy Calves and Their Relationship with Clinical Status, Lung Consolidation, and Average Daily Gain. J. Vet. Intern. Med. 2015, 29, 381-387. [CrossRef]

40. Klima, C.L.; Alexander, T.W.; Hendrick, S.; McAllister, T.A. Characterization of Mannheimia haemolytica Isolated from Feedlot Cattle That Were Healthy or Treated for Bovine Respiratory Disease. Can. J. Vet. Res. 2014, 78, 38-45. [CrossRef] 